\title{
Metastatic non-muscle invasive bladder cancer with cervical lymph node metastasis
}

Pablo Garrido-Abad ${ }^{1}$, Luis García Martín ${ }^{1}$, Karen Villar Zarra ${ }^{2}$, Ariel Díaz Menéndez ${ }^{2}$, Manuel Fernández Arjona ${ }^{1}$

${ }^{1}$ Department of Urology, Hospital Universitario del Henares, Coslada, Universidad Francisco de Vitoria, Madrid, Spain; ${ }^{2}$ Department of Pathology, Hospital Universitario del Henares, Coslada, Universidad Francisco de Vitoria, Madrid, Spain

\section{ABSTRACT}

Bladder cancer is a common cancer that may present as superficial, invasive, or metastatic disease. Non-muscle-invasive bladder cancer (NMIBC) represents the majority of bladder cancer diagnoses, but represents a spectrum of disease with a variable clinical course, notably for significant risk of recurrence and potential for progression. NMIBC metastasis to distant organs without local invasion or regional metastasis is a very rare occurrence, so there are limited case reports about early metastasis in the literature.

\section{ARTICLE INFO}

Pablo Garrido-Abad

http://orcid.org/0000-0003-2734-9387

Keywords:

Urinary Bladder Neoplasms;

Neoplasm Metastasis; Lower

Urinary Tract Symptoms

Int Braz J Urol. 2019; 45: 1270-4

Submitted for publication:

December 21, 2018

Accepted after revision:

June 16, 2019

Published as Ahead of Print:

August 20, 2019

\section{CASE REPORT}

A 72-year-old man with diabetes was admitted in January 2017 with complaints of lower urinary tract symptoms and microscopic hematuria. Review of the medical history showed that in 2012 he had undergone transurethral resection of a low-grade pTa bladder tumor (TURBT), without signs of recurrence until February 2017 when atypical urothelial cells were described by urine cytology and a small superficial bladder neoplasm was found on cystoscopy. Hence, the patient was treated again with TURBT. At this time the pathological diagnosis was secondary carcinoma in situ (CIS) (Figures 1A-D), that was confirmed by an additional pathologist review, so he was treated with $81 \mathrm{mg}$ of intravesical bacillus Calmette-Guerin (BCG) once weekly for 6 weeks+3 weekly instillations every 3 months. Following the protocol of high-risk bladder cancer, upper urinary tract (UUT) was evaluated with computed tomography (CT) urography without signs of UUT 
Figure 1 - Histopathology of the bladder after TURBT: (A) H-E stain (20x) with high-grade urothelial CIS. (B) Strong full thickness expression of cytokeratin 20. (C) High proliferative activity measured by Ki67 index. (D) Strong and diffuse overexpression of p53 protein (positivity was seen throughout urothelial thickness). Immunochemistry after fine needle aspiration cytology of the LNM: intense positivity for CK7 (E) CK2O (F) and GATA-3 expression (G). (Magnification of 400X).

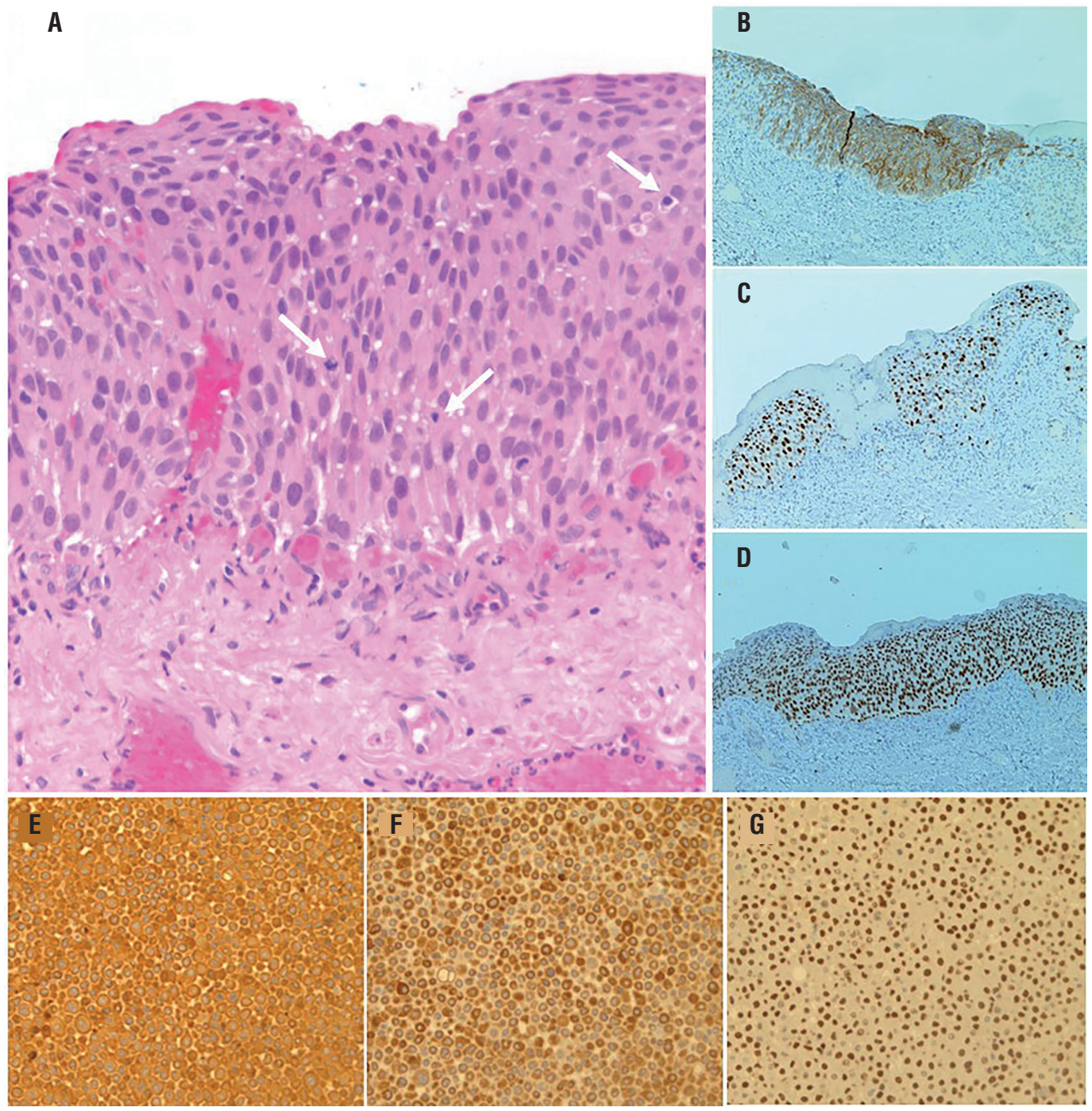

disease (Figure 2). Thus, a second TUR (Re-TUR) with random bladder biopsies was performed 45 days later, with non-tumoral bladder tissue in histopathology.

Postoperatively, the patient was followed with cystoscopy and urinary cytology every three months and remained free of recurrence for 8 months, when he complained about a left cervical mass. A cervical US (Figure 3A) and full-body CT scan (Figure 3B) revealed several left supraclavicular lymph node (LN) enlargement without evidence of local or regional (pelvic) recurrence. Fine-needle aspiration cytology (FNAC) of LNs was performed under US guidance. The immunochemical staining showed diffuse co-expression of cytokeratin 7 ( $F i-$ gure 1E), cytokeratin 20 (Figure 1F) and GATA-3 (Figure 1G), with negative expression of TTF1 and napsin A, that definitely identified metastatic UC. He received 6 cycles of combination chemotherapy (gemcitabine-cisplatin) every 4 weeks, with a 
Figure 2 - (A, B) Coronal and (C) axial scans of computed tomography (CT) urography without signs of UUT disease.
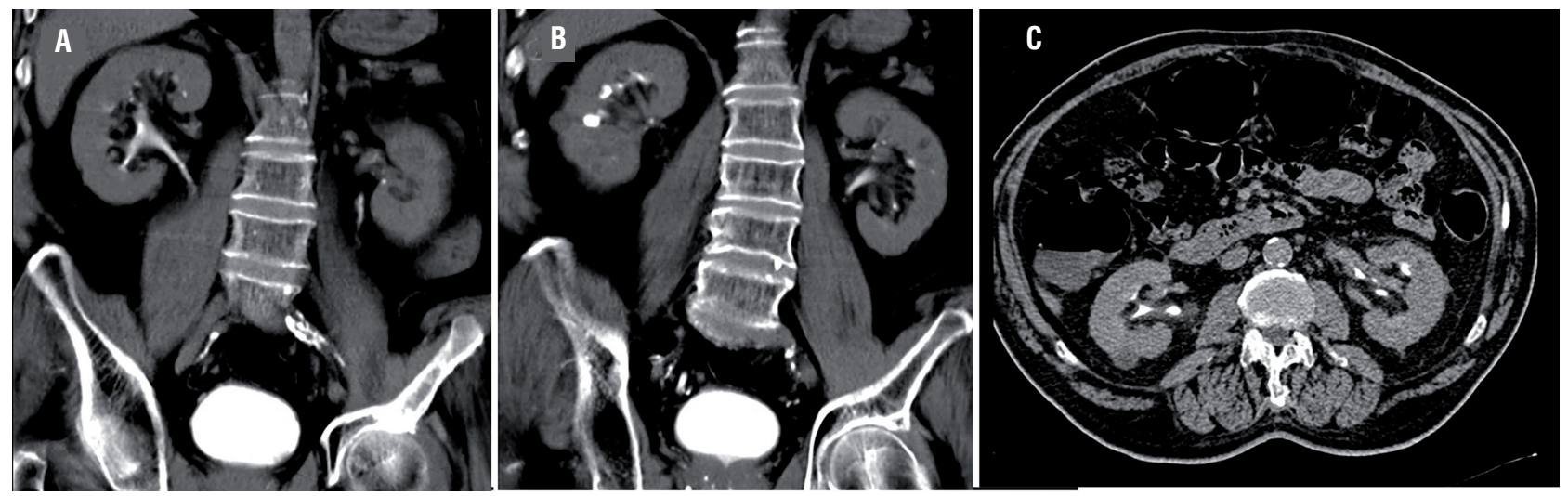

Figure 3 - (A) Ultrasound of the left supraclavicular region and (B) contrast-enhanced computed tomography scan showing enlarged left supraclavicular LNs (arrows).
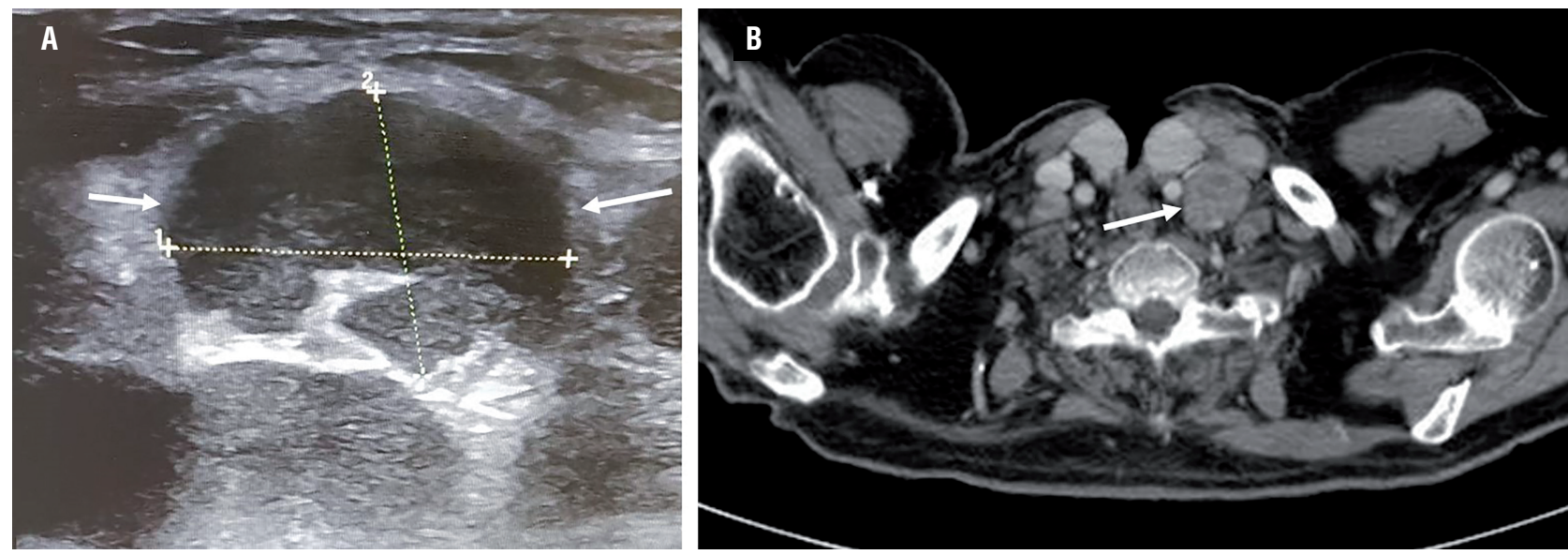

progressive clinical worsening, complicated by a fatal acute respiratory failure 5 months later.

\section{DISCUSSION}

Bladder cancer $(\mathrm{BC})$ is the seventh most commonly diagnosed cancer in the male population worldwide (1). Approximately $75 \%$ of patients with $\mathrm{BC}$ present with a disease confined to the mucosa (Ta, CIS), or submucosa (T1), being the urothelial carcinoma (UC), the most frequent histological subtype (2). NMIBC prognosis is based on the pathologic findings regarding tumor grade, multiplicity, size, concomitant CIS, depth of invasion, and early recurrence, but are not adequately accurate in predicting the individual clinical behavior (3). TURBT is the initial critical step in the management and staging of disease. An inadequate resection of the initial tumor (inadequate sampling of muscularis propria or missing tumors such as CIS) can lead to an improper staging and early recurrences (4). Half of all NMIBC patients will experience tumor recurrence within 5 years, and $20-30 \%$ will progress to secondary muscle-invasive bladder cancer (5). Ultimately, as many as $10-15 \%$ of patients presenting with NMIBC will die of bladder cancer (6), with progression-free 5 -year survival rate of $77 \%$ and an overall survival rate of 63\% after 5 years (7).

CIS of the bladder is a flat, high-grade, non-invasive UC comprising about $10 \%$ of all cases of BC. The management of CIS continues to 
present with many challenges, although intravesical BCG has been accepted as first-line therapy, with overall response rate of $86.6 \%$ and a 5-year progression-free survival rate of $78.5 \%$ (8), this can be complicated by recurrence and progression. Recent study showed that $12 \%$ of CIS had a regional LN invasion after radical cystectomy (9), so EAU guidelines also recommend radical cystectomy (10), mainly in patients with BCG refractory high risk NMIBC, rapid relapsing or refractory disease ( $<6$ months), T1 disease on repeat transurethral resection, high-volume multifocal high-grade disease, presence of lymphovascular invasion with $\mathrm{T} 1$ disease, unfavorable or mixed histology, and poor patient compliance, because they are at significant risk of progression and metastasis (11, 12). The current role of early cystectomy in CIS is controversial, some altered gene expression as high mRNA expression of ERBB2 is suggested to be useful to identify a high-risk group for progression who will benefit from an early cystectomy (13), although the potential for overtreatment must also be considered.

This is a case of initially diagnosed NMIBC that developed early, multiple cervical metastases. It is very unusual for a patient with NMIBC to develop head and neck metastasis without regional LNM or local invasion. It is difficult to establish the exact lymphatic pathways to the neck nodes from primary tumors arising below the clavicles, although there is a predilection for such metastases to be located to the left neck, while involvement of right-sided neck nodes may be associated with more extensive mediastinal involvement. Rarely, cancers originating from sites other than head and neck can metastasize to the cervical LN chain. However, genitourinary tract tumors, make up a significant proportion of these cancers and should be considered in the differential diagnosis of metastatic lesions of the head and neck (14). CIS metastases to distant organs without local invasion or regional metastases are a very rare occurrence, so there are limited case reports in the literature $(3,15,16)$.

Because of the low cost and high accuracy, the combination of US and FNAC represents a valuable and reliable method of choice for diagnosis in most cases of enlarged LNs from an unk- nown primary (17). Immunohistochemical as well as molecular studies can render a more accurate tissue diagnosis and can be carried out on cytology samples, being GATA-3 the most helpful immune stain for UC (17). Attempts to improve LN staging have shifted imaging towards increased use of MRI, which has a better soft tissue resolution (being able to detect LNM in normal sized LNs) than conventional CT (18). Routine use of F-fluorodeoxyglucose (18F-FDG) positron emission tomography (PET)/CT is still not recommended in bladder cancer staging or in LNM detection (18), however, its use is becoming more common due to recent reports suggesting that PET/CT and SUVmax interpretation is an appropriate tool in the evaluation of LN and can be used prior to surgery to make a safe identification of positive LNs (PET provides information on the potential malignancy of a LN through glucose metabolism by $18 \mathrm{~F}-\mathrm{FDG}$ uptake) (19).

Metastatic $B C$ remains an aggressive disease associated with limited treatment options and reduced survival. As many as $20 \%$ of patients will ultimately die of metastatic disease, and a significant proportion will develop both intravesical and extravesical recurrences (4), being LNs, bones, and lung the three most common sites of metastasis.

Standard treatment for metastatic cervical LN BC is not well established due to few cases reported, however, palliative radiation therapy and chemotherapy has been offered (19). There is no current reported evidence to support therapeutic neck dissection for the management of $\mathrm{BC}$ metastases to the neck $(17,20)$.

Some biomarkers, like p53 and Ki-67, are well studied and found to be correlated with progression in BC (21). Meanwhile, diabetic patients with NMIBC present more early distant metastases than expected, although they are non-invasive (22). For all this, high-risk patients with marked alterations in combined biomarker expression may benefit more from immediate radical cystectomy.

\section{CONFLICT OF INTEREST}

None declared. 


\section{REFERENCES}

1. Ferlay J, Soerjomataram I, Dikshit R, Eser S, Mathers C, Rebelo $\mathrm{M}$, et al. Cancer incidence and mortality worldwide: sources, methods and major patterns in GLOBOCAN 2012. Int J Cancer. 2015;136:E359-86.

2. Babjuk M, Burger M, Comperat E. Non-muscle invasive bladder cancer. EAU guidelines. 2018. Available at. <https:// uroweb.org/wp-content/uploads/Guidelines_WebVersion_ Complete-1.pdf>

3. Hong $\mathrm{JH}$. Early isolated bone metastases without local recurrence in non-muscle invasive bladder cancer. Int J Surg Case Rep. 2015;10:41-4.

4. Woldu SL, Bagrodia A, Lotan Y. Guideline of guidelines: nonmuscle-invasive bladder cancer. BJU Int. 2017;119:371-80.

5. Chamie K, Litwin MS, Bassett JC, Daskivich TJ, Lai J, Hanley JM, et al. Recurrence of high-risk bladder cancer: a population-based analysis. Cancer. 2013;119:3219-27.

6. van den Bosch S, Alfred Witjes J. Long-term cancer-specific survival in patients with high-risk, non-muscle-invasive bladder cancer and tumour progression: a systematic review. Eur Urol. 2011;60:493-500.

7. May M, Helke C, Nitzke T, Vogler H, Hoschke B. Survival rates after radical cystectomy according to tumor stage of bladder carcinoma at first presentation. Urol Int. 2004;72:103-11.

8. Takenaka A, Yamada Y, Miyake H, Hara I, Fujisawa M. Clinical outcomes of bacillus Calmette-Guérin instillation therapy for carcinoma in situ of urinary bladder. Int J Urol. 2008;15:30913.

9. Bruins HM, Skinner EC, Dorin RP, Ahmadi $H$, Djaladat $H$, Miranda $G$, et al. Incidence and location of lymph node metastases in patients undergoing radical cystectomy for clinical non-muscle invasive bladder cancer: results from a prospective lymph node mapping study. Urol Oncol. 2014;32:24.e13-9.

10. Babjuk M, Böhle A, Burger M, Capoun O, Cohen D, Compérat EM, et al. EAU Guidelines on Non-Muscle-invasive Urothelial Carcinoma of the Bladder: Update 2016. Eur Urol. 2017;71:447-61.

11. Monteiro LL, Witjes JA, Agarwal PK, Anderson CB, Bivalacqua TJ, Bochner BH, et al. ICUD-SIU International Consultation on Bladder Cancer 2017: management of non-muscle invasive bladder cancer. World $\mathrm{J}$ Urol. 2019;37:51-60.

12. Chang SS, Cookson MS. Radical cystectomy for bladder cancer: the case for early intervention. Urol Clin North Am. 2005;32:147-55.
13. Breyer J, Otto W, Wirtz RM, Wullich B, Keck B, Erben P, et al. ERBB2 Expression as Potential Risk-Stratification for Early Cystectomy in Patients with pT1 Bladder Cancer and Concomitant Carcinoma in situ. Urol Int. 2017;98:282-9.

14. Ogunyemi 0 , Rojas A, Hematpour K, Rogers D, Head C, Bennett C. Metastasis of genitourinary tumors to the head and neck region. Eur Arch Otorhinolaryngol. 2010;267:273-9.

15. Teyssonneau D, Daste A, Dousset V, Hoepffner JL, Ravaud A, Gross-Goupil M. Metastatic non-muscle invasive bladder cancer with meningeal carcinomatosis: case report of an unexpected response. BMC Cancer. 2017;17:323.

16. Sasaki Y, Oi H, Oyama T, Kagawa J, Komori M, Senzaki T, et al. [Non-muscle invasive bladder cancer with multiple bone metastasis without local invasion : a case report]. Hinyokika Kiyo. 2013;59:669-72.

17. López F, Rodrigo JP, Silver CE, Haigentz M Jr, Bishop JA, Strojan $P$, et al. Cervical lymph node metastases from remote primary tumor sites. Head Neck. 2016;38 Suppl 1:E2374-85.

18. Salminen AP, Jambor I, Syvänen KT, Boström PJ. Lymph node imaging in bladder cancer. Minerva Urol Nefrol. 2015;18. [Epub ahead of print]

19. Vind-Kezunovic S, Bouchelouche K, Ipsen P, Høyer S, Bell C, Bjerggaard Jensen J. Detection of Lymph Node Metastasis in Patients with Bladder Cancer using Maximum Standardised Uptake Value and (18)F-fluorodeoxyglucose Positron Emission Tomography/Computed Tomography: Results from a High-volume Centre Including Long-term Follow-up. Eur Urol Focus. 2019;5:90-6.

20. Sengeløv L, Kamby C, von der Maase H. Pattern of metastases in relation to characteristics of primary tumor and treatment in patients with disseminated urothelial carcinoma. J Urol. 1996;155:111-4.

21. Jakse G, Hall R, Bono A, Höltl W, Carpentier P, Spaander JP, et al. Intravesical BCG in patients with carcinoma in situ of the urinary bladder: long-term results of EORTC GU Group phase II protocol 30861. Eur Urol. 2001;40:144-50.

22. Ozer K, Horsanali MO, Gorgel SN, Ozbek E. Diabetes is an Important Risk Factor for Metastasis in Non- Muscle-Invasive Bladder Cancer. Asian Pac J Cancer Prev. 2016;17:105-8.

Correspondence address: Pablo Garrido-Abad, MD.

Urology Department, Hospital Universitario del Henares 28822

Avda: Marie Curie s.n. Coslada, Madrid, Spain

Phone: 0034911212200

E-mail:pgabad@hotmail.com 